\title{
Impact on Uro-oncology
}

This area is important and relevant to the NHS for a number of reasons. There is an increasing number of prostate cancer survivors within around the world. A service with a high level of patient care, focused on psychosexual concerns, side effects of therapy whilst clinically safe and based on patient need should be provided. Current follow-up systems for this cohort have a significant financial burden associated. There are significant issues with erectile dysfunction and side effects of therapy. There is a lack of available guidance for this cohort of survivors. Current follow-up programmes in the NHS are not meeting patient needs. The implications for the urooncology community are: development of guidelines for Psychosexual Survivorship Care, applicable nationally and internationally. 